\title{
Écrire sa voix vers le sacré : Kerouac face à la frontière
}

\author{
Sarah-Danièle Bélanger \\ Université de Toronto
}

La littérature, comme type de production discursive qui offre du « je », de la subjectivité, de l'intériorité à foison, constitue un espace de mise en scène et de performance qui met en jeu ce que Kierkegaard appelle la primitivité, soit le possible de l'esprit, et fournit ainsi l'espace spirituel (et matériel) d'un rapport à soi, d'une réappropriation de l'intériorité. Au-delà des catégories liées au genre (fiction, autofiction, autobiographie), la primitivité, c'est-à-dire le soi et son possible - sous-entendu le soi et la possibilité de sa transformation, conçue par 
Kierkegaard comme une reprise - ne se manifeste que dans la singularité du «je », dont l'auteur souligne l'importance en ces mots dans La Dialectique de la communication, un texte programmatique inachevé :

C'est à la personnalité que nous devons atteindre. Et je me fais un mérite d'avoir, en introduisant des personnalités fictives (mes pseudonymes) qui disent «Je» au milieu de la vie réelle, contribué dans la mesure du possible à habituer mes contemporains à réentendre parler un Je, un Je personnel (et non pas ce Je pur et ventriloque, fruit de l'imagination). Or c'est justement parce que l'évolution du monde s'est poursuivie le plus loin possible de cette reconnaissance de la personnalité qu'il fallait agir sur le plan de la fiction. Une personnalité poétique est toujours mieux acceptée par un monde déshabitué à entendre parler un Je. Et sans doute n'irai-je jamais plus loin. Je ne m'enhardirai sans doute jamais à user directement de mon propre Je. Mais il est une chose dont je suis sûr : le temps viendra où, dans le monde, s'élèvera un Je qui dira tout bonnement «Je » et parlera à la première personne. Aussi bien sera-t-il le premier à communiquer au sens le plus strict la vérité éthique et éthico-religieuse. (p. 62-63)

Le « je » kerouacquien s'offre précisément dans l'esprit de cette recherche d'une vérité éthico-religieuse. On pourrait avancer que toute son œuvre (sa Légende des Duluoz, son journal et ses lettres, ses cahiers de notes sur ses lectures bouddhistes, ses poèmes et ses haïkus) se produit comme la quête d'une conversion, comme epistrophè et metanoïa, et communique précisément par et à travers cet élan.

Le mot «conversion», du latin conversio, qui signifie simplement «retournement» ou "changement de direction», correspond en fait, comme le mentionne Hadot dans Exercices spirituels et philosophie antique, à deux mots grecs de sens différents: "d'une part epistrophè qui signifie "changement 
d'orientation", et implique l'idée d'un retour (retour à l'origine, retour à soi), d'autre part, metanoia qui signifie "changement de pensée", "repentir", et implique l'idée d'une mutation et d'une renaissance » (p. 223). Loin d'être univoque, le terme renvoie donc à deux sens différents, voire opposés, et instaure une tension entre fidélité et rupture. La frontière entre ces deux sens du mot conversion demeure floue puisque, la plupart du temps, l'expérience se produit comme un assemblage, toujours particulier, entre une forme de fidélité ou d'un retour à soi et une rupture transfiguratrice (comme arrachement à l'aliénation ou au péché). La séparation entre ces deux sens de conversion n'existe donc, le plus souvent, que comme rapprochement ou conjugaison. Ils cohabitent et se complètent à l'intérieur d'un concept qui n'existe que comme paradoxe. L'étymologie du terme s'accroche à l'expérience qu'il recouvre et la littérature - entre autres comme dialectique écriture / lecture - recueille toutes sortes de traces de cette expérience, allant même jusqu'à la provoquer.

L'écriture de Kerouac, en particulier, ne fait pas que témoigner d'une quête de conversion, elle la prépare et la performe également. C'est-à-dire que son œuvre retient les traces d'un exercice spirituel accompli sur des décennies et s'inscrit dans ce que Kierkegaard appelle une communication de pouvoir qui, par le moyen d'un «je» singulier entre autres, renvoie le possible - le possible de l'esprit ou le possible dans sa dimension spirituelle - dans le champ du lecteur. D'ailleurs, dans ce contexte, le mot « œuvre » n'est pas tout à fait approprié, car il fige le texte dans une tradition et renvoie sa réception à une lecture critique qui peut s'avérer sclérosante. L'expression "soulwork», que Kerouac lui-même utilise dans ses journaux pour parler de ses romans, exprime parfaitement la nature de cette écriture spirituelle. Il décrit l'expression en ces termes : 
A « soulwork » instead of a «novel », although of course such a name is too fancy, and laughable, but it does indicate someone's writing all-out for the sake of earnestness and salvation. The idea is that such a work must infold the man like his one undeniable cloak and dream of things... his « vision of the world and of the proposition of things », say. (Kerouac, 2004, p. 95)

Le verbe «infold», dans ce contexte, exprime quelque chose d'intéressant: on y saisit l'idée d'un enveloppement lié à l'esprit, qui provient de l'intimité et est réintégré par l'intimité, comme sérieux et désir de salut. Un tel travail littéraire est l'âme telle que mise en scène dans l'écriture et représente en même temps une forme d'exercice spirituel (un travail de l'âme sur elle-même). C'est en ce sens qu'il produit un effet d'enveloppement dirigé vers l'intériorité qui, comme communication de pouvoir, se répercute jusqu'au lecteur. D’ailleurs, l'image de la cape (cloak) qu'il utilise rappelle celle de l'habit ou du manteau textuel proposée par Thoreau dans son Walden, qui évoque l'idée d'une expérience exemplaire (au sens où elle parle) offerte pour recouvrir, exprimer ou abriter celle d'un lecteur. Pour Thoreau, la littérature livre des récits qui nous sont indirectement adressés et qui ont le pouvoir d'exprimer quelque chose de nous et de nous transformer. Il exprime une vision proprement spirituelle de la lecture en écrivant :

There are probably words addressed to our condition exactly, which, if we could really hear and understand, would be more salutary than the morning or the spring to our lives, and possibly put a new aspect on the face of things for us. How many a man has dated a new era in his life from the reading of a book! The book exists for us, perchance, which will explain our miracles and reveal new ones. The at present unutterable things we may find somewhere uttered. The same questions that disturb and puzzle and confound us have in their turn occurred to all the wise men; not one has been omitted; and each has answered them, according to his ability, by his words and his life. (p. 102) 
Ce passage de Thoreau concerne, en un sens, la communication de pouvoir kierkegaardienne. Un texte peut nous écouter dans l'indicible que nous transportons et ainsi contribuer à nous révéler à nous-mêmes. Ce manteau du texte, cette cape que représente le "soulwork», tout extérieur qu'il soit, permet au lecteur de s'envelopper et de se retourner vers une intériorité désormais contenue, protégée de la dissémination par l'habit qui lui convient.

L'expression que choisit Kerouac pour parler de ses livres - cette idée de la littérature comme "travail de l'âme »décrit parfaitement l'exigence spirituelle qu'il adresse à la littérature comme écrivain et comme lecteur. «Writing all-out » ou «reading all-in» représentent les deux faces complémentaires de la communication indirecte, de la communication de pouvoir telle que la conçoit Kierkegaard. Dans ces quelques mots, «soulwork», «writing all-out», Kerouac exprime l'essentiel de sa vision littéraire et spirituelle. Par l'écriture spontanée, l'écrivain déploie son «je» de la manière la plus brute, sans aucun calcul, comme un mouvement de l'âme qui s'exerce à se saisir, à se reprendre, à se révéler à soi-même. Dans Desolation Angels, un texte assez tardif, il réfléchit sur cette «méthode» de prose spontanée où s'imbriquent sa vision littéraire et sa vision spirituelle ancrées dans une intimité qu'il exprime à travers le surnom de son enfance, Ti Jean :

I was originating (without knowing it, you say?) a new way of writing about life, no fiction, no craft, no revising afterthoughts, the heartbreaking discipline of the veritable fire ordeal where you can't go back but have made the vow of «speak now or forever hold your tongue " and all of it innocent go-ahead confession, the discipline of making the mind the slave of the tongue with no chance to lie or re-elaborate (in keeping not 
only with the dictums of Dichtung Warheit Goethe but those of the Catholic Church my childhood) - I wrote those manuscripts as I'm writing this one in cheap nickel notebooks by candlelight in poverty and fame - Fame of self - For I was Ti Jean, and the difficulty in explaining all this and «Ti Jean» too is that readers who havent read up to this point in the earlier works are not filled in on the background - The background being my brother Gerard who said things to me before he died, though I dont remember a word, or maybe a few (I was only four) - But said things to me about a reverence for life, no, at least a reverence of the idea of life, which I translated as meaning that life itself is the Holy Ghost - . (p. 256)

Ce passage est très chargé. Les dimensions existentielle, spirituelle et littéraire s'entremêlent avec une réelle cohérence intime où on perçoit toute l'exigence éthico-religieuse de sa démarche, qui tire son origine d'un élan de révérence pour la vie dont il place lui-même la source dans le souvenir de son frère Gérard, présenté littéralement comme un saint (dans Visions of Gerard). Cet élan le pousse à écrire à partir de ce qui pour lui est sacré et à écrire dans ses cahiers cheap par cette parole que la pensée ne contrôle plus, c'est-à-dire par ce mouvement, cet élan vital, existentiel, spirituel, dont la pensée est devenue l'esclave qu'il décrit dans ses «Essentials of Spontaneous Prose » :

The best writing is always the most painful personal wrungout tossed from cradle warm protective mind - tap from yourself the song of yourself, Blow! - now! - your way is your only way - « good» - or « bad» - always honest, («ludicrous»), spontaneous, « confessional» interesting, because not crafted. Craft is craft.

Kerouac s'est exercé à écrire pendant des années pour en venir à cette méthode d'écriture qui, finalement, rejette l'écriture ouvrée, travaillée de façon minutieuse, comme une pièce d'artisanat ou comme mise en scène. Il la rejette selon cet idéal 
d'une confession innocente, soufflée en bloc, soustraite à l'esprit organisateur qui, d'habitude, orchestre le discours sur soi. L'esprit n'est plus cette barrière (ou ce berceau) qui protège, il est ce dont le souffle ou le beat se répand dans un «soulwork» qui n'admet pas les limites de la grammaire et qui semble rejeter la médiation, du moins la médiation temporelle, celle de la pensée après-coup. Une écriture qui, à travers la performance, se veut une traversée des frontières de l'esprit organisateur et qui cherche à se produire comme éveil de l'esprit. Dans Some of the Dharma, Kerouac écrit: "ANY WRITING THAT IS NOT SELF-ENLIGHTENING WILL ROT LIKE A BODY. If I write THE GOLDEN BOOK OF DULUOZ every line will have to be self-enlightening » (p. 278). Il n'y a d'écriture sacrée - et d'éternité du texte que dans l'éveil à soi d'un "soulwork», un éveil à soi qui se comprend selon le paradigme de la conversion à travers le paradoxe même de son étymologie, soit comme epistrophè (retour à l'origine) et comme metanoïa (changement de pensée).

L'écriture de Kerouac ne se saisit pas entièrement selon le discours identitaire habituel lié à l'autofiction, précisément parce que son enjeu n'est pas identitaire mais bien spirituel. Le "soulwork» produit dans une prose spontanée se rapporte en effet entièrement au registre spirituel et implique une véritable reconfiguration de la conception de la production littéraire vers le paradigme de la conversion. Cette nuance entre les dimensions identitaire et spirituelle dans l'écriture peut être comprise à travers le différend qu'ont connu Foucault et Hadot sur la question de l'écriture de soi à travers l'exemple des carnets de notes spirituels antiques (hupomnêmata). Foucault utilise l'exemple de ces carnets de notes spirituels, où les stoïciens et les épicuriens consignent leurs pensées en relation 
avec un savoir qu'ils recueillent du même coup, pour réfléchir à la visée et aux implications de ce qu'il appelle «l'écriture de soi ». Pour lui, l'écriture - de soi - est un mode de constitution du soi en relation avec un savoir, un logos ou une sagesse conçus et accumulés sur un mode horizontal. L'écriture de soi appartient au vaste ensemble des modes et des pratiques de subjectivation et d'assujettissement identifiés et interprétés par Foucault à partir d'une vision du savoir essentiellement immanente. Le rejet de la transcendance dans ce rapport au savoir ne permet de penser la conversion que dans la perspective identitaire de la constitution ou reconstitution du soi. Chez Hadot, au contraire, la verticalité domine la conception du rapport qui s'établit entre le soi, le savoir et l'écriture et conduit au final à détacher l'écriture - même quand elle porte sur soi-même - de la subjectivité. Pour Hadot, "non seulement on ne s'écrit pas soi-même, mais l'écriture ne constitue pas le soi : [...] elle fait changer le moi de niveau, elle l'universalise » (p. 329). C'est-à-dire qu'à travers l'exemple des hupomnêmata (de même que celui des confessions chrétiennes), Hadot considère que le rôle de l'écriture est d'allier les dimensions universelle et particulière, soit d'élever le particulier à l'universel et également, comme le dirait Kierkegaard, de faire parler l'universel dans le particulier.

Malgré les apparences, Kerouac ne s'écrit pas lui-même. En mettant l'accent sur le langage, sur le rythme, sur le flux spontané, il sort du registre de la mimésis, ce qui empêche de parler d'écriture de soi. Il n'écrit pas non plus vraiment pour lui-même. L'exigence spirituelle d'écrire pour soi implique toujours en même temps d'écrire dans le regard de l'autre, d'une collectivité ou de Dieu (c'est là l'idée même d'une écriture comme confession qui revient à plusieurs reprises dans ses 
textes). Il écrit à travers lui-même, en direction de lui-même et de l'universel. En ce sens, on peut considérer que la plupart de ses textes sont des carnets de notes spirituels (peu importe leur statut sur le plan de la fiction). Some of the Dharma en particulier semble très proche-dans l'esprit comme dans le résultat-des hupomnêmata antiques sur lesquels se sont penchés Foucault et Hadot. Il s'agit véritablement d'un cahier spirituel composé de ses notes et réflexions sur le bouddhisme. Son titre, qui est un jeu de mots sur l'homophonie de «some » et «sum », indique déjà une réflexion sur la partie et le tout, sur le particulier et l'universel, sur la possible traversée de l'un à l'autre ou l'interpénétration de l'un et de l'autre, qu'il mène à partir des bribes d'un savoir assemblées et entremêlées à ses propres pensées sur lui-même, sur son écriture. Ce carnet de notes, comme travail préparatoire à la conversion, est le véritable travail, c'est-à-dire qu'il incarne, en lui-même, sa propre finalité spirituelle. Comme le jeu de mots autour de la partie et du tout l'indique, et comme Hadot le souligne dans ses références aux hupomnêmata antiques, ce type d'écriture est en réalité « un exercice par lequel le moi se situe dans la totalité et s'éprouve comme partie de cette totalité » (p. 310). L'écriture agit comme mouvement de reprise et de dépassement du soi. Si, dans cette perspective, Some of the Dharma s'avère exemplaire, cette fonction et cette visée de l'écriture caractérisent l'œuvre de Kerouac de manière générale. Sa prose, sa poésie et ses journaux sont tous également, d'une certaine façon, des carnets de notes spirituels qu'on ne peut comprendre qu'à partir du paradigme de la conversion. La première entrée du journal de 1948 de Kerouac est représentative de cette fonction de l'écriture comme pratique spirituelle. Il note: «Thus - my new diary begins. And its purpose, simply, to rediscover my real 
voice which is yours too, all our real, one voice, that's so often drowned by criticism and fear » (2004, p. 155). Il ne s'agit pas de se découvrir - l'écriture de Kerouac ne se produit pas dans l'esprit du « souci de soi » et du processus de subjectivation que trouve Foucault dans l'écriture comme exercice spirituel-, il s'agit véritablement de se reprendre, de redécouvrir sa propre voix dans son écho universel, et donc, paradoxalement peutêtre, de s'approcher au plus près du soi en s'extirpant des frontières qui l'entourent, le subjectivisent et le maintiennent du même coup dans son petit tassement. En ce sens, la pratique de la prose spontanée et son corollaire - le rejet du «craft», de l'écriture travaillée - permettent de traverser les frontières déterminées d'une conscience organisatrice et d'accéder à une forme de singularité de laquelle s'exprime l'universel (epistrophè), ou encore transfigurée par l'universel (metanoïa).

La notion de «soulwork» exprime bien l'idée de cette écriture comme exercice spirituel qui vise l'universel dans le particulier et comme exercice de conversion, c'est-à-dire, littéralement, comme le paradoxe ou la conjugaison d'un mouvement d'epistrophè et de metanö̈a. Le déploiement du soi sous forme d'assemblage de souvenirs dans sa Légende de Duluoz, le «soulwork» de sa vie, n'est que le premier plan d'une démarche plus profonde qui vise l'éveil à soi et la rédemption. Ce qu'il appelle le «self-enlightement» et que doit viser et incarner l'écriture, le "soulwork» lui-même, concerne le soi précisément dans sa dimension universelle. Cette forme d'éclairage qui émane de l'intériorité tout en dépassant les frontières de la subjectivité, qui a le pouvoir d'irradier selon un plan vertical, universel, à partir d'un ancrage particulier, est une autre façon d'exprimer la communication de pouvoir que devrait mettre en jeu l'écriture. Le «self-enlightement», la rédemption ou la conversion représentent 
l'impulsion à la source de la communication de pouvoir où, à travers et par-delà le texte, écrivain et lecteur se placent face à leur propre existence et se constituent en "universels singuliers", pour reprendre l'expression de Kierkegaard, expression qui signifie que sa préoccupation pour sa propre intériorité ne se cloisonne pas aux frontières du particulier, mais qu'elle contient une signification universelle grâce à son rapport au possible qu'est le religieux. L' ' universel singulier » comme catégorie spirituelle et littéraire, rappelle ce que semblait chercher à exprimer Thoreau dans le passage de son Walden cité au début de cet article : «The same questions that disturb and puzzle and confound us have in their turn occurred to all the wise men; not one has been omitted; and each has answered them, according to his ability, by his words and his life» (p.102). Même le ratage spirituel de l'écriture contribue au déploiement de cet « universel singulier » et participe de la communication de pouvoir par laquelle se passent et se partagent les mêmes questions. Un ratage que Kerouac exprime à la toute fin de Vanity of Duluoz en reprenant à son compte un passage de L'Ecclésiaste :

I settled down to write, in solitude, in pain, writing hymns and prayers even at dawn, thinking "When this book is finished, which is going to be the sum and substance and crap of everything I've been thru throughout this whole goddam life, I shall be redeemed. »

But, wifey, I did it all, I wrote the book, I stalked the streets of life, of Manhattan, of Long Island, stalked thru 1,183 pages of my first novel, sold the book, got an advance, whooped, hallelujah'd, went on, did everything you're supposed to do in life.

But nothing ever came out of it. No « generation » is «new ». There's «nothing new under the sun ». «All is vanity». (p. 268) 
Le paradigme de la conversion au $\mathrm{XX}^{\mathrm{e}}$ siècle ne se conçoit plus en fonction de sa réussite, mais de son élan, une autre façon de dire que la logique de la question et l'affect qui l'accompagne priment sur celle de la réponse (et du savoir institutionnel qui la fonde).

\section{Bibliographie}

FouCAULT, Michel. (1994 [1980-1988]), Dits et écrits, t. IV, Paris, Gallimard, coll. « Bibliothèque des sciences humaines ».

HADOT, Pierre. (2002), Exercices spirituels et philosophie antique, Paris, Albin Michel.

KerouaC, Jack. (1993 [1965]), Desolation Angels, New York, Riverhead Books.

-. (1957) "Essentials of Spontaneous Prose», $<$ http://www.writing.upenn.edu/ afilreis/88/kerouacspontaneous.html>.

-. (2004), Windblown World. The Journals of Jack Kerouac 1947-1954, Londres / New York, Penguin Books.

-. (1997), Some of the Dharma, Londres / New York, Penguin Books.

-. (1994 [1968]), Vanity of Duluoz: An Adventurous Education, 1935-46, Londres, New York, Penguin Books.

KieRKEgAARD, Søren. (2004), la Dialectique de la communication, Paris, Payot \& Rivages.

Thoreau, Henry David. (2000 [1854]), Walden and Other Writings, New York, The Modern Library. 


\title{
Résumé
}

Cet article se propose d'explorer la pratique spirituelle kerouacquienne de l'écriture, qui s'impose comme tentative de dépasser la médiation participant normalement de toute écriture, qu'elle soit littéraire ou non, à travers une discipline qui vise à faire de l'esprit et de la main qui écrit les esclaves d'une voix intérieure projetée sans retour sur soi dans un mouvement qui vise essentiellement la conversion.

\begin{abstract}
This paper intends to reflect on Kerouac's spiritual writing practice, which appears as an attempt at overcoming the mediation that normally belongs to writing (may it be of a literary nature or not). It argues that, through his writing discipline making the mind and the writing hand the slaves of a projected inner voice, his writing impulse and ethics essentially aim for conversion.
\end{abstract}

